\title{
Store-Brand Production Arrangement Based on the Game Theory
}

\author{
Weimin Ma, ${ }^{1}$ Rong Cheng $\left(\mathbb{D},{ }^{1}\right.$ Hua Ke, ${ }^{1}$ and Jianguang Zhang ${ }^{2}$ \\ ${ }^{1}$ School of Economics and Management, Tongji University, Shanghai 200092, China \\ ${ }^{2}$ Business School, Guilin University of Electronic Technology, Guilin 541004, China \\ Correspondence should be addressed to Rong Cheng; 13667737255@163.com
}

Received 10 June 2017; Revised 1 November 2017; Accepted 27 February 2018; Published 1 April 2018

Academic Editor: Luis M. López-Ochoa

Copyright (c) 2018 Weimin Ma et al. This is an open access article distributed under the Creative Commons Attribution License, which permits unrestricted use, distribution, and reproduction in any medium, provided the original work is properly cited.

\begin{abstract}
The increasing popularity of store brands is resulting in greater cannibalization of national brands. Thus, national-brand manufacturers are trying their best to confront this trend. At the same time, however, many leading national-brand manufacturers have become involved in the store-brand production of their counterpart retailers. We construct a game-theory-based framework to model the strategic interaction between a leading national-brand manufacturer and a retailer. Besides the national brand, the retailer also has an option for its own store brand to compete with the national brand head to head. There are two choices for the store-brand production available to the retailer: a fringe manufacturer with low production efficiency or alternatively the nationalbrand manufacturer with high efficiency. It is shown that, under certain conditions, there is a win-win situation for both the storebrand retailer and the national-brand manufacturer with the latter supplying the store brand. More interestingly, it is found that the national-brand manufacturer supplying the store brand may lead to a higher likelihood of the store brand introduction. Our study offers an explanation for why more and more national-brand manufacturers supply store brands.
\end{abstract}

\section{Introduction}

Store-brand (SB) (also named private label or own brand) products are merchandise sold under a retailer's brand name and play a significant role in the differentiation and profitability of the retailing business. According to the latest data from the Private Label Manufacturers Association, SB volume share was nearly $25 \%$ in the USA [1], $46 \%$ in the $\mathrm{UK}$, and increased in 13 of the 20 Europe countries [2]. Due to the successful expansion of SBs and tough brand competition between SB products and national-brand (NB) products, this area has attracted abundant research interests. However, most of the research regarding SB focuses on the strategies and impacts of the SB introduction. Few researchers have paid attention to the SB production issue [3]. In the real business practice, there are three general classifications of the manufacturers producing SB products: large manufacturers who produce both their own NBs and SBs; small and medium manufacturers that specialise in particular product lines and concentrate on producing SBs almost exclusively; and major retailers and wholesalers that operate their own manufacturing plants and provide SBs for their own stores [2].

It is shown that retailers' outsourcing SB production to NB manufacturers prevails in the real world, and more than $50 \%$ of US NB manufacturers of consumer packaged goods made SBs as well and provided more than $60 \%$ of SBs (by volume) $[4,5]$. Since more and more NB manufacturers have been engaging in SB production, increasing studies are of interest in the rationale for why NB manufacturers are willing to provide their retailers with SBs that compete with their own NBs head to head. After all, SBs would cannibalize NBs. However, most of the related studies only investigate the issue from NB manufacturers' points of view to investigate why and when a NB manufacturer would produce SB for its counterpart retailer. On the other hand, why a SB retailer accepts the same is far from clear, which is an important question proposed by Sethuraman and Raju [6]. Some other studies try to answer this question under the framework of bargaining model. However, the business practice wherein 
profits are divided in the form of wholesale prices and retail markups is fairly common in the real world. To address this problem when profits are divided by pricing strategy instead of bargaining model, we use a Stackelberg-game model to shed light on the strategic interaction on SB production arrangement between a NB manufacturer and a retailer with SB introduction option. By solving the game and analyzing the equilibrium results, we find that under certain conditions, both the SB retailer and the NB manufacturer are better off with the latter supplying the store brand, and the NB manufacturer supplying SB may lead to a higher likelihood of the SB introduction. Therefore, we help to fill the gap of the extant research and obtain some new managerial insights on the SB production arrangement issue.

The remainder of this paper is organized as follows. We review some related studies in Section 2. Section 3 makes some assumptions and formulates our model. Section 4 solves games and obtains equilibrium outcomes. Section 5 makes comparisons and analyzes our main equilibrium and the conditions. Accordingly, we highlight the main results and propose corresponding managerial implications in Section 6. In Section 7, we discuss and offer suggestions for the future research.

\section{Literature Review}

In the literature, there are a few analytical models attempting to explain the phenomenon that NB manufactures supply SB for their retailers. Tarziján [7] assumes that producing SB can generate economies of scale and a high perceived SB quality, whereby the NB manufacturer can foreclose SB produced by any other independent manufacturer. However, his study only looks at the issue from NB manufacturer's point of view. Departing from Tarziján [7], both Bergès-Sennou [8] and Bergès and Bouamra-Mechemache [9] assume that the NB manufacturer supplying SB can strengthen its bargaining power on NB vis-à-vis the retailer; meanwhile the retailer is also better off with a lower SB supply cost from the NB manufacturer. The profits from $\mathrm{NB}$ and $\mathrm{SB}$ products are ultimately divided between the NB manufacturer and the retailer by the bargaining model. Obviously, the conclusions of Bergès-Sennou [8] and Bergès and Bouamra-Mechemache [9] may not correspond to the business practices that profits are divided in the form of wholesale prices and retail markups. More recently, Hara and Matsubayashi [10] analytically demonstrate that a premium SB, instead of a low perceived-quality $\mathrm{SB}$, can facilitate the production collaboration between retailers and NB manufacturers. As a matter of fact, low perceived-quality SBs, also known as copycats of NBs, account for the overwhelming majority of SBs in the real market. Meanwhile, leading NB manufacturers producing low perceived-quality SBs are not uncommon [2]. In addition, some other studies also provide interesting insights into this SB production issue. Empirically, Littman [11] and Ter Braak et al. [12] show that, by supplying SBs for retailers, NB manufacturers can cultivate a better relationship with their retailers. Wu and Wang [13] reveal that a NB manufacturer supplying SB can mitigate promotional competition with another NB manufacturer. Gomez-Arias and Bello-Acebron
[14] investigate the SB production arrangement between a high-quality NB manufacturer and a low-quality one, concluding that all game players profit from the arrangement with the former supplying the premium store brand and the latter otherwise. Chambolle et al. [15] demonstrate that outsourcing the SB production to the NB manufacturers can spur innovation on NB and avoid the NB exclusion from the retailer's shelves. Yano et al. [16] find that a retailer should sell its SB factory to a third-party producer under certain conditions, and both the retailer and the NB manufacturer would benefit from this. To improve the SB product quality, Mai et al. [17] investigate what kind of extended warranty contracts with the SB producer are most effective. Distinguished from the aforementioned studies, we look at the issue from the perspectives of both NB manufacturers and retailers and investigate whether and when they both have incentives to collaborate with a low perceived-quality SB production issue, where the profits are divided in the form of wholesale prices and retail markups. By focusing on the interaction between a NB manufacturer and a retailer with the SB introduction option, we show that there is a win-win situation with SB being produced by the NB manufacturer.

This article is also related to the literature on the coordination between NB manufacturers and SB retailers. Gabrielsen and Sørgard [18] claim that the NB manufacturer can foreclose the SB introduction by offering an exclusivity contract on the NB wholesale price. On the contrary, Fang et al. [19] argue that when NB cost per unit quality is larger than that of SB, the NB manufacturer is unable to deter the retailer from introducing $\mathrm{SB}$. They propose a simple minimum order quantity contract to coordinate the supply chain. Groznik and Heese [20] reveal that only a longterm contract on the NB wholesale price can provide the NB manufacturer with the commitment ability to prevent a retailer from introducing a store brand. Chen et al. [21] show that the retailer's capability of developing SB is a critical factor that determines the SB's role in the performance of the whole supply chain. Amrouche and Yan [22] examine the effect of $\mathrm{NB}$ advertising and revenue sharing strategies on all channel members as well as the whole channel. More recently, Zhang et al. [23] show that the manufacturer and retailer may be better off cooperatively sharing the forecast information and generating a consensus forecast. Different from previous research, our paper investigates whether a NB manufacturer and a $\mathrm{SB}$ retailer can collaborate on the arrangement of the SB production.

Game theory is often employed to study many kinds of supply chain management problems. He et al. [24] utilize differential game theory to analyze cooperative advertising in a multiple-manufacturer single-retailer supply chain. Both He et al. [25] and Zhang et al. [26] demonstrate advertisement strategy based on game theory. In a simple supply chain with a manufacturer and its supplier, Xu et al. [27] study opportunistic behavior in the vertical $R \& D$ cooperation between the two supply chain members by utilizing game theory. Facing a sensitive demand and based on the multiperiod dynamic game theory, Zhou and Ju [28] analyze the reputation effect and ratchet effect in the multiperiod dynamic game service supply chain. Similarly to our paper, both Li et al. [29] and 
Shi et al. [30] use Stackelberg-game theory. However, Li et al. [29] investigate impacts of risk aversion of supply chain players on price and quality decisions. On the other hand, Shi et al. [30] study different coordination performances of different contracts in a closed-loop supply chain system. Interestingly, Malmir et al. [31] use game theory to model the strategies of supplying medical equipment in a hospital supply chain. By means of evolutionary game theory, Feng et al. [32] study cooperative mechanism in a supply chain of prefabricated construction and find that there is a winwin situation. Likewise, we also utilize game theory to study supply chain problem. However, we focus on another special supply chain (i.e., the supply chain without SB), which will contribute to this stream of research.

\section{Model}

Consider a channel wherein a NB manufacturer (he) sells his product through a local monopolist retailer (she) and the retailer has the motivation and capability to introduce her SB. Initially, the NB manufacturer produces NB of a particular category at a constant production cost, $c_{n}$, and wholesales it to his retailer at the wholesale price, $w_{n}$. In turn, the retailer resells $\mathrm{NB}$ to the end consumers at retail price, $p_{n}$. We denote the equilibrium outcomes in this case with superscript 0 . Since the NB production cost is constant and would not influence the manufacturer's optimal decision, we set $c_{n}$ equal to zero without loss of generality. Then, we allow the retailer to introduce her SB within the same category and if so, distribute $\mathrm{SB}$ at retail price, $p_{s}$, alongside with $\mathrm{NB}$. As a consequence, the retailer has to decide how to outsource the SB production. She has two choices: a competitive fringe from a perfectly competitive market (the equilibrium outcomes in this case are denoted with superscript $f$ ) and the NB manufacturer (denoted with superscript $m$ ). As for the same product quality, in line with many other studies $[8,9,33]$, we assume the NB manufacturer has a comparative advantage in production efficiency relative to the competitive fringe. It is believable that an experienced NB manufacturer can possess a technology or services difference compared with the independent fringe manufacturer when producing a SB $[34,35]$; economies of scale may be an additional reason for this advantage [7]. The NB manufacturer can offer SB for the retailer with a constant price, $w_{m}$, and incur a production cost equal to that of the $\mathrm{NB}$, due to the same production process and product quality; that is, $c_{s}^{m}=c_{n}=0$. Alternatively, the retailer also can entrust her SB production to the competitive fringe with a constant purchasing price, $w_{f}$. Since the fringe manufacturer comes from a perfectly competitive market, it acts as a dumb player and makes no margin at all. Additionally, due to a lower efficiency compared with the NB manufacturer, $w_{f}$ is assumed to be equal to his production cost, $c_{s}^{f}$ and $c_{s}^{f}>c_{n}=0$.

Within this particular category, consumers make purchasing decisions to maximize their utilities by comparing the prices and quality of the products. We assume that consumers are heterogeneous in the valuation of every unit perceived quality and denote this value by $v$. For analytic simplicity, $v$ is assumed to be uniformly distributed within the consumer population from 0 to 1 with unit density. Each consumer is assumed to buy at most one unit of either NB or SB product within the same category. Although both NB and SB are produced in the same quality, consumers deem SB as an inferior substitute for $\mathrm{NB}$ as a result of heavy NB advertisements [36-39]. In other words, consumers perceive a higher quality of NB than SB. We normalize the perceived quality of $\mathrm{NB}$, and the SB perceived quality is assumed to $\gamma(0<\gamma<1)$. Therefore, the valuation of NB is $v$ and SB is $\gamma v$. Given retail prices $p_{n}$ and $p_{s}$, the SB perceived quality $\gamma$, and the consumer's valuation $v$ of the unit perceived quality, the consumer derives a utility of $v-p_{n}$ by buying $\mathrm{NB}$ and $\gamma v-p_{s}$ by buying SB. As a result, the consumers with the valuation $v \geq p_{n}$ would consider buying NB. Equivalently, the consumers with the valuation $v \geq p_{s}$ would consider buying SB. If $v-p_{n}>\gamma v-p_{s}$, then the consumer would prefer to buy NB over SB. Consequently, there are two kinds of marginal consumers. One refers to those whose valuation equals $\left(p_{n}-p_{s}\right) /(1-\gamma)$ and are indifferent between the two brands; the other one refers to those whose valuation equals $p_{s} / \gamma$ and are indifferent between buying SB and buying nothing. If $p_{s}>\gamma p_{n}$, then SB would never be bought. So the demands for the two brands, respectively, are

$$
\begin{aligned}
& q_{n}= \begin{cases}1-\frac{P_{n}-P_{s}}{1-\gamma} & \text { if } p_{s}<\gamma p_{n}, \\
1-p_{n} & \text { otherwise, }\end{cases} \\
& q_{s}= \begin{cases}\frac{\gamma P_{n}-P_{s}}{\gamma(1-\gamma)} & \text { if } p_{s}<\gamma p_{n}, \\
0 & \text { otherwise. }\end{cases}
\end{aligned}
$$

We consider the game where the NB manufacturer is in a dominant position vis-à-vis the retailer and acts as a Stakerberg-game leader. We assume that the competitive fringe is a nonstrategic player and takes $w_{s}$ as an exogenous variable. Thus, there are two stages of the game when the retailer has option for SB introduction:

(i) Stage 1: the manufacturer, as a price leader, firstly announces his wholesale price, $w_{n}$, for his NB and leaves a take-it-or-leave-it contract to the retailer.

(ii) Stage 2: given the NB wholesale price, $w_{n}$, the retailer as a follower determines whether or not to introduce $\mathrm{SB}$ by maximizing her profit. If she decides to introduce her SB, she should simultaneously decide who supplies SB for her and the retail prices of both brands.

All notations used in our model are summarized in the Notations.

\section{Equilibrium Market Outcomes}

4.1. NB Only. We first consider the benchmark case where the NB manufacturer distributes his NB through the retailer, who acts as a local monopolist. In this setting, the retailer only sells the NB and has no option for her SB. Hence, the consumers with the valuation no less than $p_{n}$ would buy NB, 
TABLE 1: Equilibrium outcomes with NB only.

\begin{tabular}{ccccc}
\hline$w_{n}^{0}$ & $p_{n}^{0}$ & $q_{n}^{0}$ & $\pi_{r}^{0}$ & $\pi_{m}^{0}$ \\
\hline$\frac{1}{2}$ & $\frac{3}{4}$ & $\frac{1}{4}$ & $\frac{1}{16}$ & $\frac{1}{8}$ \\
\hline
\end{tabular}

and the demand is $q_{n}=1-p_{n}$. The corresponding profits of the NB manufacturer and the retailer are $\pi_{m}=w_{n}\left(1-p_{n}\right)$ and $\pi_{r}=\left(p_{n}-w_{n}\right)\left(1-p_{n}\right)$, respectively. The NB manufacturer first determines the wholesale price $w_{n}$, and then the retailer sets the retail price $p_{n}$. We solve this game with backwards induction. Thus, for the given wholesale price $w_{n}$, the retailer's best response is $p_{n}=\left(w_{n}+1\right) / 2$. Anticipating the retailer's best response function, the manufacturer determines his wholesale price $w_{n}$ to maximize his profits $\pi_{m}=w_{n}(1-$ $\left.w_{n}\right) / 2$, which yields the optimal wholesale price $w_{n}^{0}=1 / 2$. The equilibrium profits of the manufacturer and the retailer, respectively, are $\pi_{m}^{0}=1 / 8$ and $\pi_{r}^{0}=1 / 16$. We summarize the equilibrium outcomes in Table 1.

4.2. With SB Supplied by the Competitive Fringe. Now, we allow the retailer to have the option for introducing SB. She can choose a fringe supplier from a perfectly competitive market to supply her SB at the price $w_{f}$ equal to the production cost. In this case, the NB manufacturer firstly sets a wholesale price $w_{n}$ for his $\mathrm{NB}$, and, given $w_{n}$, the retailer decides whether or not to introduce her SB, and subsequently prices NB and SB, if SB is introduced. Likewise, we solve this problem backwards. Firstly, we suppose the retailer decided to introduce her SB and purchased it from the competitive fringe at price $w_{f}$, and then her profits by distributing both $\mathrm{SB}$ and $\mathrm{NB}$ are

$$
\pi_{r}=\left(p_{n}-w_{n}\right)\left(1-\frac{p_{n}-p_{s}}{1-\gamma}\right)+\left(p_{s}-w_{f}\right) \frac{\gamma p_{n}-p_{s}}{\gamma(1-\gamma)} .
$$

For given $w_{n}$, the retailer maximizes her profits with respect to $p_{n}$ and $p_{s}$, respectively, yielding the optimal prices

$$
\begin{aligned}
& p_{n}=\frac{1+w_{n}}{2}, \\
& p_{s}=\frac{\gamma+w_{f}}{2} .
\end{aligned}
$$

Anticipating the retailer's reaction, the NB manufacturer chooses his wholesale price to maximize his profits

$$
\pi_{m}=w_{n}\left(1-\frac{p_{n}-p_{s}}{1-\gamma}\right)
$$

Solving this problem, we can obtain the equilibrium prices and profits of the game as follows:

$$
\begin{aligned}
& w_{n}^{f}=\frac{1-\gamma+w_{f}}{2} ; \\
& p_{n}^{f}=\frac{3-\gamma+w_{f}}{4}, \\
& p_{s}^{f}=\frac{\gamma+w_{f}}{2} ;
\end{aligned}
$$

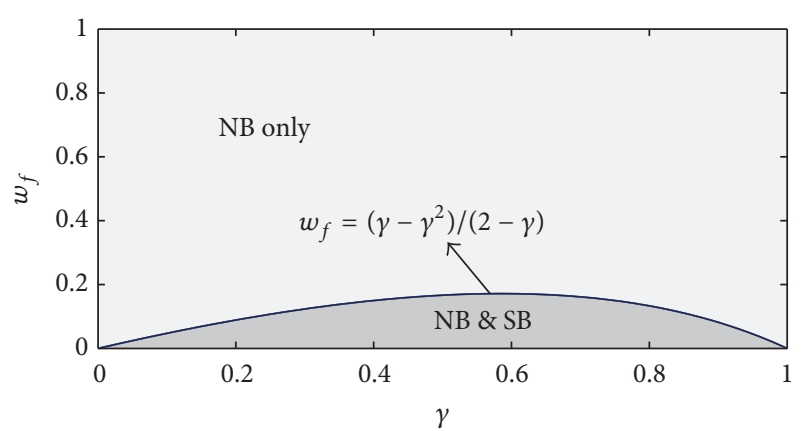

FIgURE 1: Retailer's SB entry strategy with SB supplied by the competitive fringe.

$$
\begin{aligned}
\pi_{r}^{f} & =\frac{\gamma+2 \gamma^{2}-3 \gamma^{3}+6 \gamma^{2} w_{f}-3 \gamma w_{f}-6 \gamma w_{s}+4 w_{f}^{2}}{16 \gamma(1-\gamma)}, \\
\pi_{m}^{f} & =\frac{\left(1-\gamma+w_{f}\right)^{2}}{8(1-\gamma)} .
\end{aligned}
$$

To ensure the nonnegative demand for SB, the constraint $p_{s}^{f}<\gamma p_{n}^{f}$ must hold, and then we derive that

$$
w_{f}<\frac{\gamma-\gamma^{2}}{2-\gamma}
$$

Next, by comparing the retailer's profits with and without $\mathrm{SB}$, we can get the retailer's decision on the SB introduction conditional on the SB perceived quality $\gamma$ and the SB supply cost $w_{f}$. That is, when $w_{f}<\left(\gamma-\gamma^{2}\right) /(2-\gamma)$, the retailer will always introduce her SB. Otherwise, she will not. We characterize decisions of the retailer and the NB manufacturer as follows.

Proposition 1. When $S B$ is produced by the competitive fringe and supplied at the price $w_{f}$ and if $w_{f}<\left(\gamma-\gamma^{2}\right) /(2-$ $\gamma)$, anticipating the retailer will always introduce her $S B$, the manufacturer will lower the NB wholesale price as $w_{n}^{f}=(1-$ $\left.\gamma+w_{f}\right) / 2$, and the resulting profits of the retailer and the manufacturer, respectively, are $\pi_{r}^{f}=\left(\gamma+2 \gamma^{2}-3 \gamma^{3}+6 \gamma^{2} w_{f}\right.$ $\left.3 \gamma w_{f}^{2}-6 \gamma w_{f}+4 w_{f}^{2}\right) / 16 \gamma(1-\gamma)$ and $\pi_{m}^{f}=\left(1-\gamma+w_{f}\right)^{2} / 8(1-\gamma)$. Otherwise, the retailer never introduces $S B$.

To illustrate Proposition 1 more clearly, we plot the retailer's SB introduction decision conditional on $\gamma$ and $w_{f}$ in Figure 1. It is easy to derive other equilibrium outcomes, as shown in Table 2.

4.3. With SB Supplied by NB Manufacturer. In this case, we assume that the NB manufacturer produces SB for the retailer and supplies it at a fixed price $w_{m}$, which satisfies $0<w_{m}<$ $w_{f}$. Otherwise, there is no incentive for the retailer to make the NB manufacturer supply SB. To find the equilibrium, we also use the backward induction. Hence, we start with the 
TABLE 2: Equilibrium outcomes with SB supplied by the competitive fringe.

\begin{tabular}{lcc}
\hline$w_{f}$ & $\left(0, \frac{\gamma(1-\gamma)}{2-\gamma}\right)$ & {$\left[\frac{\gamma(1-\gamma)}{2-\gamma}, 1\right)$} \\
\hline$w_{n}^{f}$ & $\frac{1-\gamma+w_{f}}{2}$ & $\frac{1}{2}$ \\
$p_{n}^{f}$ & $\frac{3-\gamma+w_{f}}{4}$ & $\frac{3}{4}$ \\
$p_{s}^{f}$ & $\frac{\gamma+w_{f}}{2}$ & N/A \\
$q_{n}^{f}$ & $\frac{1-\gamma+w_{f}}{4(1-\gamma)}$ & $\frac{1}{4}$ \\
$q_{s}^{f}$ & $\frac{\gamma-\gamma^{2}+\gamma w_{f}-2 w_{f}}{4 \gamma(1-\gamma)}$ & N/A \\
$\pi_{r}^{f}$ & $\frac{\gamma+2 \gamma^{2}-3 \gamma^{3}+\frac{6 \gamma^{2} w_{f}-3 \gamma w_{f}^{2}-6 \gamma w_{f}+4 w_{f}^{2}}{16 \gamma(1-\gamma)}}{\frac{\left(1-\gamma+w_{f}\right)^{2}}{8(1-\gamma)}}$ & $\frac{1}{8}$ \\
$\pi_{m}^{*}$ & $\frac{1}{8}$ & \\
\hline
\end{tabular}

retailer's decision on her retail pricing problem. In this case, the retailer's profits function becomes

$$
\pi_{r}=\left(p_{n}-w_{n}\right)\left(1-\frac{p_{n}-p_{s}}{1-\gamma}\right)+\left(p_{s}-w_{m}\right) \frac{\gamma p_{n}-p_{s}}{\gamma(1-\gamma)} .
$$

To maximize the retailer's profits, we obtain her best reaction to the given $w_{n}$ :

$$
\begin{aligned}
& p_{n}=\frac{1+w_{n}}{2}, \\
& p_{s}=\frac{\gamma+w_{m}}{2} .
\end{aligned}
$$

Different from the case with SB supplied by the competitive fringe, now the NB manufacturer's profits not only come from the sales of the NB product but also come from the SB product. Therefore, the NB manufacturer's profits function is

$$
\pi_{m}=w_{n}\left(1-\frac{p_{n}-p_{s}}{1-\gamma}\right)+w_{m} \frac{\gamma p_{n}-p_{s}}{\gamma(1-\gamma)} .
$$

Substituting the retailer's response functions into the NB manufacturer's profits function and taking first-order condition with respect to $w_{n}$, we obtain

$$
w_{n}^{m}=\frac{1-\gamma}{2}+w_{m}
$$

As a result, the equilibrium prices and profits of this game are

$$
\begin{aligned}
p_{n}^{m} & =\frac{3-\gamma+2 w_{m}}{4}, \\
p_{s}^{m} & =\frac{\gamma+w_{m}}{2} ; \\
\pi_{r}^{m} & =\frac{\gamma+3 \gamma^{2}+4 w_{m}^{2}-8 \gamma w_{m}}{16 \gamma}, \\
\pi_{m}^{m} & =\frac{\gamma-\left(\gamma-2 w_{m}\right)^{2}}{8 \gamma} .
\end{aligned}
$$

We observe that, under this pricing policy, the condition for the nonnegative SB demand (i.e., $p_{s}^{m}<\gamma p_{n}^{m}$ ) always holds. Sequentially, we check whether the retailer has the incentive to introduce her SB when supplied by the NB manufacturer at the price $w_{m}$. Comparing the retailer's profits with SB supplied by the NB manufacturer and without SB yields the condition for the SB introduction in this case as follows:

$$
w_{m}<\frac{\gamma}{2}
$$

Consequently, we obtain the retailer and the NB manufacturer's strategies in this case.

Proposition 2. When the NB manufacturer supplies $S B$ at the price $w_{m}$ and only when $w_{m}<\gamma / 2$, the retailer will introduce her $S B$, and the NB manufacturer will set his NB wholesale price as $w_{n}^{m}=(1-\gamma) / 2+w_{m}$. The equilibrium profits, respectively, are $\pi_{r}^{m}=\left(\gamma+3 \gamma^{2}+4 w_{m}^{2}-8 \gamma w_{m}\right) / 16 \gamma$ and $\pi_{m}^{m}=(\gamma-(\gamma-$ $\left.\left.2 w_{m}\right)^{2}\right) / 8 \gamma$. Otherwise, the retailer never introduces $S B$.

The SB introduction strategy is depicted in Figure 2 and all equilibrium results for this case are summarized in Table 3. It is very interesting to notice that the demand for the NB product in this case is a constant and equals that in the scenario without SB entry.

\section{Comparison and Analysis}

In this section, we first compare the conditions for the SB introduction. By comparing the equilibrium profits of both the retailer and the NB manufacturer between SB supplied by the competitive fringe and supplied by the NB manufacturer, we try to find whether both the NB manufacturer and the retailer have an incentive to make the SB product produced by the NB manufacturer. It is very straightforward to obtain the following proposition on the condition for the SB entry. 
TABLE 3: Equilibrium outcomes with SB supplied by the NB manufacturer.

\begin{tabular}{|c|c|c|}
\hline$w_{m}$ & $\left(0, \frac{\gamma}{2}\right)$ & {$\left[\frac{\gamma}{2}, 1\right)$} \\
\hline$w_{n}^{m}$ & $\begin{array}{l}\frac{1-\gamma}{2}+w_{m} \\
3-\gamma+2 w_{m}\end{array}$ & $\begin{array}{l}\frac{1}{2} \\
3\end{array}$ \\
\hline$p_{n}^{m}$ & $\begin{array}{c}4 \\
\gamma+w_{m}\end{array}$ & $\frac{5}{4}$ \\
\hline$p_{s}^{m}$ & $\frac{\gamma+1 m}{2}$ & N/A \\
\hline$q_{n}^{m}$ & $\begin{array}{c}\frac{1}{4} \\
\gamma-2 w_{m}\end{array}$ & $\frac{1}{4}$ \\
\hline$q_{s}^{m}$ & $\frac{m}{4 \gamma}$ & N/A \\
\hline$\pi_{r}^{m}$ & $\frac{\gamma+3 \gamma^{2}+4 w_{m}^{2}-8 \gamma w_{m}}{16 \gamma}$ & $\frac{1}{16}$ \\
\hline$\pi_{m}^{m}$ & $\frac{\gamma-\left(\gamma-2 w_{m}\right)^{2}}{8 \gamma}$ & $\frac{1}{8}$ \\
\hline
\end{tabular}

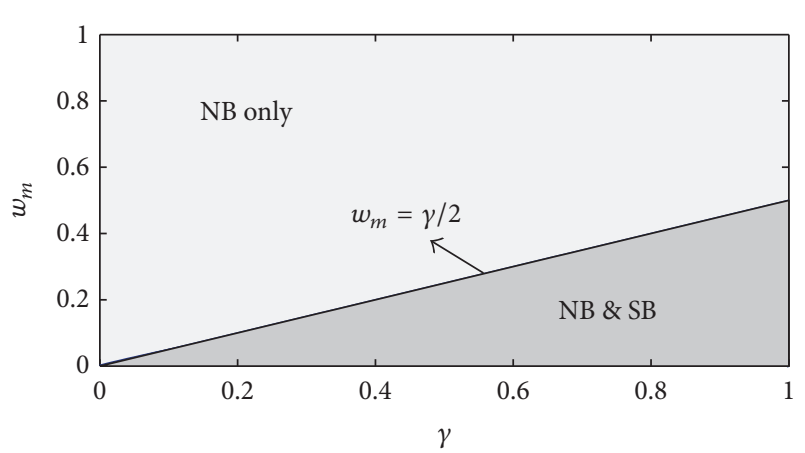

Figure 2: Retailer's SB entry strategy with SB supplied by the NB manufacturer.

Proposition 3. Compared with the case in which $S B$ is supplied by the competitive fringe, the condition for the retailer to introduce her SB might become more relaxed in the case with SB supplied by the NB manufacturer. That is,

$$
\frac{\gamma-\gamma^{2}}{2-\gamma}<\frac{\gamma}{2}
$$

To articulate Proposition 3, we plot the two conditions as a function of the SB perceived quality $\gamma$ and SB supply cost $w_{m}$ or $w_{f}$ in Figure 3. The intuition behind Proposition 3 is as follows. When a NB manufacturer supplies SB for a retailer and profits from the SB sales, he would not take SB entry as a big threat as much as when SB is supplied by the competitive fringe. This also can be reflected by his decision on the NB wholesale price. Specifically, the NB manufacturer responds to a change in the SB supply cost more heavily when he produces SB; that is, $\partial w_{n}^{m} / \partial w_{m}=1>1 / 2=$ $\partial w_{n}^{f} / \partial w_{f}$. The rationale behind this is straightforward: the SB product is not only a threat but also a source of profits for the NB manufacturer when he supplies SB. In this case, the NB manufacturer should adjust his NB wholesale price to maximize his profits from the two brands. When the $\mathrm{SB}$ supply cost is much high, the SB profitability for the

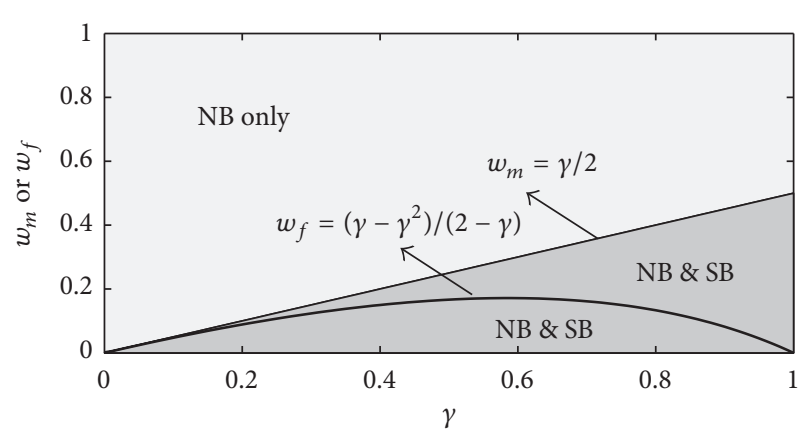

FIGURE 3: Comparison of the conditions on retailer's SB entry strategy.

retailer decreases compared with $\mathrm{NB}$, and thus the retailer may give up introducing SB. However, this is never profitable for the NB manufacturer; therefore, he will increase his NB wholesale price to the same extent as the increase in the SB supply cost, more than he does when the competitive fringe supplies SB. This means the retailer may still be willing to introduce her SB. This would definitely result in a higher likelihood of the SB entry when it is supplied by the NB manufacturer. From this point of view, the practice in which the NB manufacturer produces SB would lead SB to more likely show up on the retailer's shelves.

Next, we compare the NB manufacturer's profits to find the condition for the NB manufacturer to supply SB instead of the competitive fringe. Now that we compare the equilibria, the default premises are $w_{f}<\left(\gamma-\gamma^{2}\right) /(2-\gamma)$ and $w_{m}<\gamma / 2$. The difference in the NB manufacturer's profits between the two cases (SB is supplied, resp., by the NB manufacturer and by the competitive fringe) is given by

$$
\begin{aligned}
& \Delta \pi_{m}=\pi_{m}^{m}-\pi_{m}^{f} \\
& =\frac{4 \gamma w_{m}^{2}-4 \gamma^{2} w_{m}+2 \gamma^{2} w_{f}-\gamma w_{f}^{2}-4 w_{m}^{2}+4 \gamma w_{m}-2 \gamma w_{f}}{8 \gamma(1-\gamma)} .
\end{aligned}
$$


Deriving from expression of $\Delta \pi_{m}$, respectively, regarding $w_{f}$ and $w_{m}$, we can easily obtain $\partial \Delta \pi_{m} / \partial w_{f}<0$ and $\partial \Delta \pi_{m} / \partial w_{m}>0$. This means that if the SB supply cost of the competitive fringe increases, the difference in the NB manufacturer's profits between the two cases decreases and vice versa. If the SB supply cost of the NB manufacturer increases, this difference increases and vice versa. In other words, when the SB supply cost of the competitive fringe is relatively high, the NB manufacturer had better let the competitive fringe supply SB. But when his SB supply cost is high enough, it is more beneficial for the NB manufacturer supply SB. Similarly, after some algebra, we can obtain the condition for the NB manufacturer to supply SB himself. We characterize the NB manufacturer's strategy on the SB production in Proposition 4

Proposition 4. When the SB supply costs of the NB manufacturer and the competitive fringe satisfy the following conditions, it will be more profitable for the NB manufacturer to produce $S B$ for the retailer. That is,

$$
\begin{aligned}
\frac{\gamma}{2}-\frac{1}{2} \sqrt{\gamma^{2}-\frac{\gamma w_{f}\left(2-2 \gamma+w_{f}\right)}{1-\gamma}<} & w_{m}<\frac{\gamma}{2}, \\
0 & <w_{f}<\frac{\gamma-\gamma^{2}}{2-\gamma} .
\end{aligned}
$$

Otherwise, the NB manufacturer is better off letting the competitive fringe to produce $S B$.

Proposition 4 reveals that when the SB supply cost of the NB manufacturer is beyond a certain threshold (i.e., $\gamma / 2-$ $\left.(1 / 2) \sqrt{\gamma^{2}-\gamma w_{f}\left(2-2 \gamma+w_{f}\right) /(1-\gamma)}\right)$, the NB manufacturer would always choose to supply SB. On the other hand, when this cost is below the threshold, the NB manufacturer would benefit more from letting the competitive fringe supplying SB rather than himself. Obviously, this corresponds with the analysis on $\Delta \pi_{m}$. Now, we take the retailer's perspective to specify the SB production arrangement strategy when faced with two choices: the NB manufacturer or the competitive fringe. The difference in the retailer's profits between the two choices can be given by

$$
\Delta \pi_{r}=\pi_{r}^{m}-\pi_{r}^{f}=\frac{8 \gamma^{2} w_{m}-8 \gamma w_{m}-4 \gamma w_{m}^{2}+4 w_{m}^{2}+6 \gamma w_{f}-6 \gamma^{2} w_{f}+3 \gamma w_{f}^{2}-4 w_{f}^{2}}{16 \gamma(1-\gamma)} .
$$

We take the partial derivative to this profits' difference expression regarding $w_{f}$ and $w_{m}$, respectively, and obtain $\partial \Delta \pi_{r} / \partial w_{f}>0$ and $\partial \Delta \pi_{r} / \partial w_{m}<0$. This is quite understandable. When the SB supply cost of the competitive fringe increases, the retailer will be more inclined to have the NB manufacturer produce SB for her and vice versa. Next, we derive from this expression to obtain the condition in which the retailer chooses the NB manufacturer to produce her SB.

Proposition 5. The retailer will choose the NB manufacturer to produce $S B$ if and only if the $S B$ supply costs of the $N B$ manufacturer and the competitive fringe satisfy the following conditions:

$$
\begin{array}{r}
0<w_{m}<\gamma-\sqrt{\gamma^{2}-\frac{3 \gamma w_{f}\left(2-2 \gamma+w_{f}\right)-4 w_{f}^{2}}{4(1-\gamma)}}, \\
0<w_{f}<\frac{\gamma-\gamma^{2}}{2-\gamma} .
\end{array}
$$

Otherwise, the retailer will choose the competitive fringe to produce her $S B$.

Proposition 5 shows that only when the NB manufacturers SB supply cost is low enough, the retailer will choose the NB manufacturer to produce SB for her. Next, we try to specify whether there is a win-win situation for both the retailer and the NB manufacturer with SB supplied by the latter. By comparing the conditions in Propositions 4 and 5, we can obtain the conditions for the win-win situation as follows.

Proposition 6. There is a win-win situation for both the NB manufacturer and the retailer when the NB manufacturer and the competitive fringes $S B$ supply costs satisfy the following conditions:

$$
\begin{aligned}
& \frac{\gamma}{2}-\frac{1}{2} \sqrt{\gamma^{2}-\frac{\gamma w_{f}\left(2-2 \gamma+w_{f}\right)}{1-\gamma}} \\
& \quad<\gamma-\sqrt{\frac{\gamma^{2}-\frac{3 \gamma w_{f}\left(2-2 \gamma+w_{f}\right)-4 w_{f}^{2}}{4(1-\gamma)}}{2}}, \\
& \frac{\gamma}{2} \sqrt{\gamma^{2}-\frac{\gamma w_{f}\left(2-2 \gamma+w_{f}\right)}{1-\gamma}<w_{m}} \\
& \quad<\gamma-\sqrt{\frac{\gamma^{2}-\frac{3 \gamma w_{f}\left(2-2 \gamma+w_{f}\right)-4 w_{f}^{2}}{4(1-\gamma)}}{0}}, \\
& 0<w_{f}<\frac{\gamma-\gamma^{2}}{2-\gamma} .
\end{aligned}
$$

From Proposition 6, we find that there indeed exists a win-win situation for both the NB manufacturer and the retailer regarding the SB production arrangement. To be specific, when the NB manufacturers SB supply cost is suitable for $\mathrm{SB}$ introduction and the competitive fringes cost 
is intermediate, both the NB manufacturer and the retailer are better off with the SB production by the NB manufacturer, rather than by the competitive fringe. The results coincide with Chen et al. [40]. They empirically show that both NB manufacturers and retailers profit from adding SB and a vertically integrated supply of SB. More importantly, this finding provides a theoretical explanation for why more and more leading NB manufacturers are involved in SB production and manage the whole category for their retailers $[41,42]$.

\section{Conclusion and Managerial Implications}

Extant theoretical studies investigate the SB production issue either only from the perspective of the retailer or under the framework of bargaining model. Differently, we consider a setting where the NB manufacturer and the retailer divide the profits in form of Stakerberg game rather than bargaining model and use a game-theory-based framework to model the interaction between a NB manufacturer and its retailer, to gain a better understanding of why and when the NB manufacturer and the retailer would like to collaborate on SB production issue. Three cases are considered for comparison: NB only; with SB supplied by the competitive fringe; with $\mathrm{SB}$ supplied by NB manufacturer. By comparing the main equilibrium and the conditions, we help to fill the gap of the extant research and make the following contributions:

(i) Two different conditions for the different SB supplying strategies and the corresponding optimal pricing strategies for the NB manufacturer and the retailer are obtained.

(ii) It is found that when SB is supplied by the NB manufacturer, the retailer is more likely to introduce SB.

(iii) When profits are divided in the form of wholesale prices and retail markups, instead of the bargaining model, we find a win-win situation for both the NB manufacturer and the retailer.

Correspondingly, the immediate managerial implications of our results are as follows:

(i) For retailers, when they decide to introduce SB and the supply cost of NB manufactures is relatively lower than that of the competitive fringe, retailers are better off choosing NB manufactures as their SB suppliers, although the NB wholesale price would be much higher than the other case.

(ii) When retailers introduce SB not only to compete with $\mathrm{NB}$ but also to improve customer loyalty and brand awareness and so on, choosing NB manufactures to supply SB would lead to a higher likelihood of SB introduction ceteris paribus.

(iii) For NB manufacturers, it is profitable to supply SB but at the price of lower profits from their own NB. Consequently, it is not always better off supplying SB for their retailers. (iv) For both retailers and NB manufacturers, when the SB supply cost of NB manufactures is relatively low and that of the competitive fringe is relatively high, even in a competitive environment, both the NB manufacturer and the retailer would benefit from NB manufacturers supplying SB.

\section{Discussion}

The increasing popularity of SB products worldwide, especially in America and European countries, attracts a great number of research interests. Nevertheless, the understating of when and why SB production by the NB manufacturer is far from clear [3]. In particular, why a NB manufacturer would produce $\mathrm{SB}$, which competes face to face with its own brand and in the meantime why a SB retailer would accept the same [6] are not fully understood. Echoing with this, we investigate this problem in the study. Our results theoretically explain why there are more and more leading manufacturers producing SBs and even price and manage category products for their retailers as "Category Captains" in the real world $[41,42]$. Indeed, it may be more beneficial for the NB manufacturer to take an "if you can't beat them, join them" stand with regard to the SB introduction $[4,43]$, and undoubtedly producing SB is the most possible way to do so. Nevertheless, for simplicity and analytical tractability, we make some rigid assumptions that lead to several limitations of our study. First of all, we assume that the NB manufacturer's SB supplying cost is lower than that of the competitive fringe. In practice, manufacturers that specialise in SBs may have higher production efficiency than the NB manufacturer in some certain categories. As such, the change of the SB supplying cost could totally overturn our results. On the other hand, there is no horizontal competition at the retailing level in our model. Someone may consider the case with more than one retailer and obtain some interesting results. Furthermore, the SB quality is exogenous and assumed to be equal but perceived as inferior to NB. In business practice, it is fairly common for the retailer to strategically adjust its SB quality. Therefore, endogenizing the SB production quality may result in some interesting and new findings. Finally, Daudi et al. [44] point out that trust is of great importance for supply chain collaboration; thus it may be very interesting to model trust dynamics by utilizing game theory in the SB production collaboration between the NB manufacturer and the retailer.

\section{Notations}

$\gamma: \quad$ SB perceived quality

$w_{n}$ : NB wholesale price

$w_{m}$ : SB supply cost by the NB manufacturer

$w_{f}$ : SB supply cost by the competitive fringe

$p_{n}, p_{s}$ : Retail prices of $\mathrm{NB}$ and $\mathrm{SB}$, respectively

$q_{n}, q_{s}$ : Demands for NB and SB, respectively

$\pi_{m}: \quad$ NB manufacturer's profits

$\pi_{r}$ : Retailer's profits. 


\section{Conflicts of Interest}

The authors declare that there are no conflicts of interest regarding the publication of this paper.

\section{References}

[1] PLMA-Show, "Plma’s 2016 private label trade show," 2016, http:// plma.com/showinfo/showinfo2016.html.

[2] PLMA, "Private label today," 2016, http://www.plmainternational.com/industry-news/private-label-today.

[3] R. Sethuraman, "Assessing the external validity of analytical results from national brand and store brand competition models," Marketing Science, vol. 28, no. 4, pp. 759-781, 2009.

[4] J. A. Quelch and D. Harding, "Brands versus private labels: Fighting to win," 1996.

[5] N. Kumar et al., Private label strategy: how to meet the store brand challenge, Harvard Business Review Press, 2007.

[6] R. Sethuraman and J. S. Raju, "Private label strategies - Myths and realities," Handbook of Marketing Strategy, pp. 318-335, 2012.

[7] J. Tarziján, "Should national brand manufacturers produce private labels?" Journal of Modelling in Management, vol. 2, no. 1, pp. 56-70, 2007.

[8] F. Bergès-Sennou, "Store loyalty, bargaining power and the private label production issue," European Review of Agricultural Economics, vol. 33, no. 3, pp. 315-335, 2006.

[9] F. Bergès and Z. Bouamra-Mechemache, "Is producing a private label counterproductive for a branded manufacturer?" European Review of Agricultural Economics, vol. 39, no. 2, pp. 213239, 2012.

[10] R. Hara and N. Matsubayashi, "Premium store brand: Product development collaboration between retailers and national brand manufacturers," International Journal of Production Economics, vol. 185, pp. 128-138, 2017.

[11] M. Littman, "House brands hit home," 1992.

[12] A. Ter Braak, B. Deleersnyder, I. Geyskens, and M. G. Dekimpe, "Does private-label production by national-brand manufacturers create discounter goodwill?" International Journal of Research in Marketing, vol. 30, no. 4, pp. 343-357, 2013.

[13] C.-C. Wu and C.-J. Wang, "A positive theory of private label: A strategic role of private label in a duopoly national-brand market," Marketing Letters, vol. 16, no. 2, pp. 143-161, 2005.

[14] J. T. Gomez-Arias and L. Bello-Acebron, "Why do leading brand manufacturers supply private labels?" Journal of Business and Industrial Marketing, vol. 23, no. 4, pp. 273-278, 2008.

[15] C. Chambolle, C. Christin, and G. Meunier, "Optimal Production Channel for Private Labels: Too Much or Too Little Innovation?" Journal of Economics \& Management Strategy, vol. 24, no. 2, pp. 348-368, 2015.

[16] C. A. Yano, E. J. Durango-Cohen, and L. Wagman, "Outsourcing in place: Should a retailer sell its store-brand factory?" IISE Transactions, vol. 49, no. 4, pp. 442-459, 2017.

[17] D. T. Mai, T. Liu, M. D. Morris, and S. Sun, "Quality coordination with extended warranty for store-brand products," European Journal of Operational Research, vol. 256, no. 2, pp. 524-532, 2017.

[18] T. S. Gabrielsen and L. Sørgard, "Private labels, price rivalry, and public policy," European Economic Review, vol. 51, no. 2, pp. 403-424, 2007.
[19] X. Fang, S. Gavirneni, and V. R. Rao, "Supply chains in the presence of store brands," European Journal of Operational Research, vol. 224, no. 2, pp. 392-403, 2013.

[20] A. Groznik and H. S. Heese, "Supply chain conflict due to store brands: The value of wholesale price commitment in a retail supply chain," Decision Sciences, vol. 41, no. 2, pp. 203-230, 2010.

[21] L. Chen, S. M. Gilbert, and Y. Xia, "Private labels: Facilitators or impediments to supply chain coordination," Decision Sciences, vol. 42, no. 3, pp. 689-720, 2011.

[22] N. Amrouche and R. Yan, "Aggressive or partnership strategy: Which choice is better for the national brand?" International Journal of Production Economics, vol. 166, pp. 50-63, 2015.

[23] T. Zhang, X. Zhu, and Q. Gou, "Demand Forecasting and Pricing Decision with the Entry of Store Brand under Various Information Sharing Scenarios," Asia-Pacific Journal of Operational Research, vol. 34, no. 2, Article ID 1740018, 2017.

[24] Y. He, Q. Gou, C. Wu, and X. Yue, "Cooperative advertising in a supply chain with horizontal competition," Mathematical Problems in Engineering, vol. 2013, Article ID 607184, 16 pages, 2013.

[25] Y. He, Z. Liu, and K. Usman, "Coordination of cooperative advertising in a two-period fashion and textiles supply chain," Mathematical Problems in Engineering, vol. 2014, Article ID 356726, 10 pages, 2014.

[26] G. Zhang, Q. Sui, J. Hu, Y. Zhong, and H. Sun, "Equilibrium Model of Discrete Dynamic Supply Chain Network with Random Demand and Advertisement Strategy," Mathematical Problems in Engineering, vol. 2014, Article ID 539768, 2014.

[27] L. Xu, D. Liang, Z. Duan, and X. Xiao, "Stability analysis of r\&d cooperation in a supply chain," Mathematical Problems in Engineering, vol. 2015, Article ID 409286, 10 pages, 2015.

[28] G. Zhou and C. Ju, "Effect analysis of service supply chain with dynamic game under the condition of sensitive demand," Mathematical Problems in Engineering, vol. 2015, Article ID 278094, 2015.

[29] Q. Li, Z. Liu, and Y. He, "Impact of risk aversion on price and quality decisions under demand uncertainty via the CARA utility function," Mathematical Problems in Engineering, vol. 2014, Article ID 490121, 2014.

[30] Z. Shi, N. Wang, T. Jia, and H. Chen, "Reverse Revenue Sharing Contract versus Two-Part Tariff Contract under a Closed-Loop Supply Chain System," Mathematical Problems in Engineering, vol. 2016, Article ID 5464570, 2016.

[31] B. Malmir, S. Dehghani, F. F. Jahantigh, and M. Najjartabar, "A new model for supply chain quality management of hospital medical equipment through game theory," in Proceedings of the 6th International Conference on Information Systems, Logistics and Supply Chain, ILS 2016, June 2016.

[32] T. Feng, S. Tai, C. Sun, and Q. Man, "Study on Cooperative Mechanism of Prefabricated Producers Based on Evolutionary Game Theory," Mathematical Problems in Engineering, vol. 2017, Article ID 1676045, 2017.

[33] P. Bontems, S. Monier-Dilhan, and V. Réquillart, "Strategic effects of private labels. European Review of Agricultural Economics," European Review of Agriculture Economics, vol. 26, no. 2, pp. 147-165, 1999.

[34] W. S. Comanor and P. Rey, "Vertical restraints and the market power of large distributors," Review of Industrial Organization, vol. 17, no. 2, pp. 135-153, 2000.

[35] G. Galizzi, L. Venturini, and S. Boccaletti, "Vertical relationships and dual branding strategies in the Italian food industry," Agribusiness, vol. 13, no. 2, pp. 185-195, 1997. 
[36] Y. Chen, "Paying customers to switch," Journal of Economics \& Management Strategy, vol. 6, no. 4, pp. 877-897, 1997.

[37] N. Mehta, X. J. Chen, and O. Narasimhan, "Informing, transforming, and persuading: Disentangling the multiple effects of advertising on brand choice decisions," Marketing Science, vol. 27, no. 3, pp. 334-355, 2008.

[38] J.-B. E. M. Steenkamp and I. Geyskens, "Manufacturer and retailer strategies to impact store brand share: Global integration, local adaptation, and worldwide learning," Marketing Science, vol. 33, no. 1, pp. 6-26, 2014.

[39] R. Sethuraman and K. Gielens, "Determinants of store brand share," Journal of Retailing, vol. 90, no. 2, pp. 141-153, 2014.

[40] J. Chen, O. Narasimhan, G. John, and T. Dhar, "An empirical investigation of private label supply by national label producers," Marketing Science, vol. 29, no. 4, pp. 738-755, 2010.

[41] R. L. Steiner, "Category management-a pervasive, new vertical/horizontal format," Antitrust, vol. 15, p. 77, 2000.

[42] R. L. Steiner, "The nature and benefits of national brand/private label competition," Review of Industrial Organization, vol. 24, no. 2, pp. 105-127, 2004.

[43] D. Dunne and C. Narasimhan, "The new appeal of private labels," Harvard Business Review, vol. 77, no. 3, pp. 41-48, 1999.

[44] M. Daudi, J. B. Hauge, and K.-D. Thoben, "On analysis of trust dynamics in supply chain collaboration," in Proceedings of the 6th International Conference on Information Systems, Logistics and Supply Chain, ILS 2016, June 2016. 


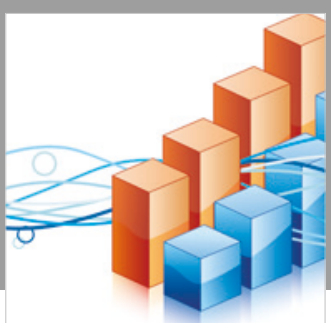

Advances in

Operations Research

\section{-n-m}
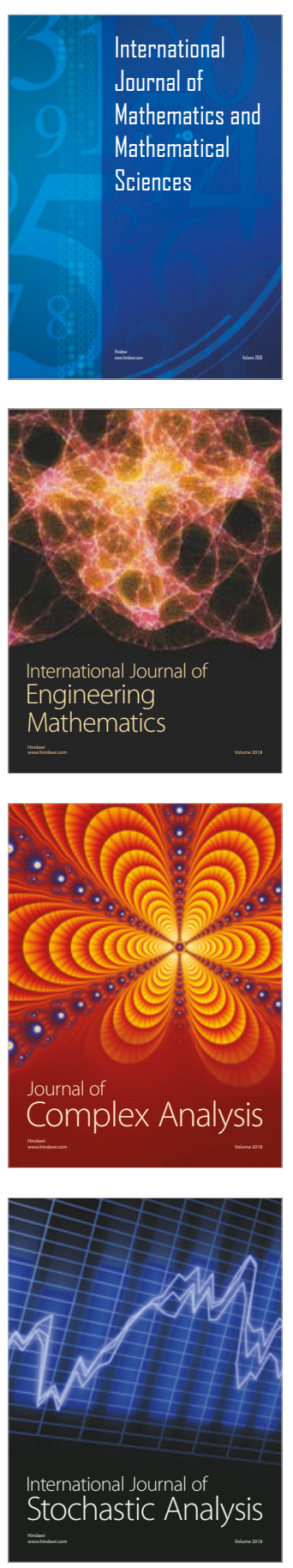
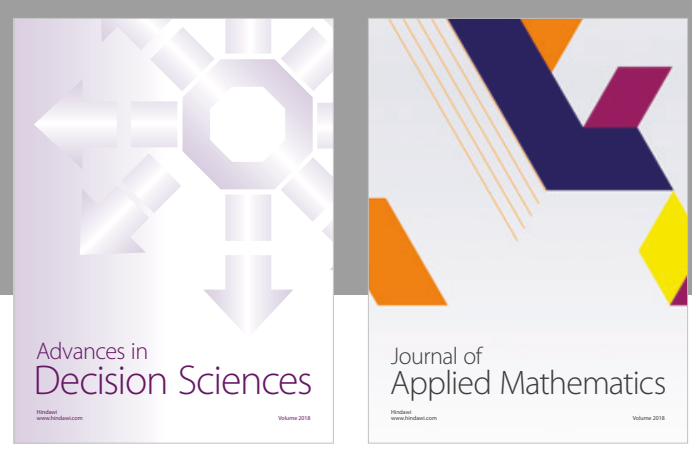

Journal of

Applied Mathematics
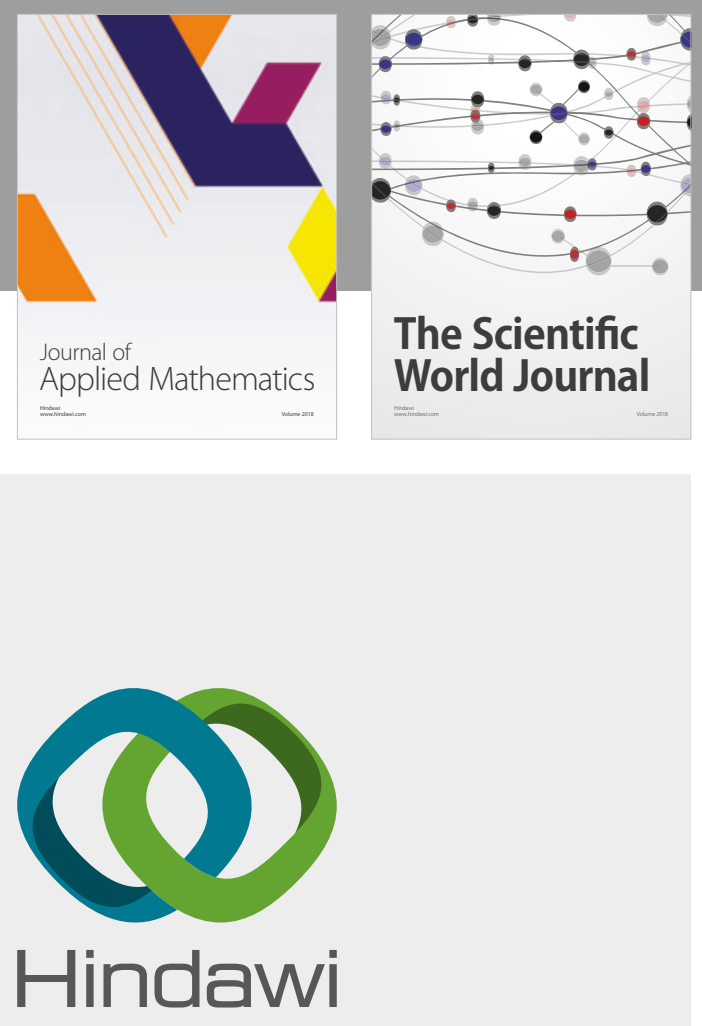

Submit your manuscripts at

www.hindawi.com

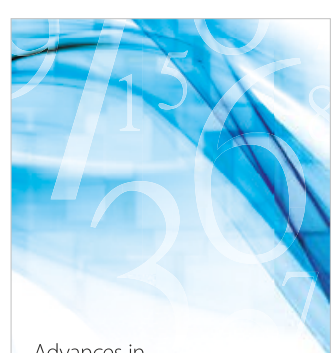

Advances in
Numerical Analysis
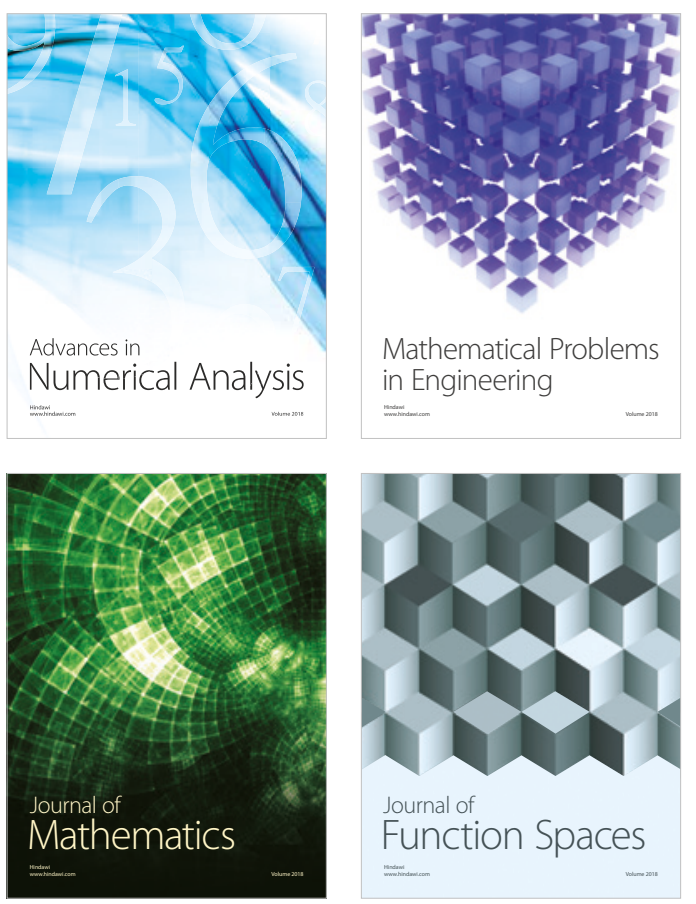

Mathematical Problems in Engineering

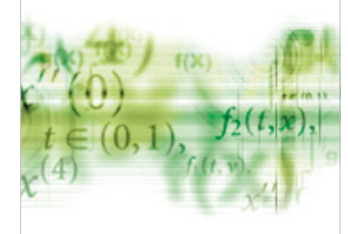

International Journal of

Differential Equations

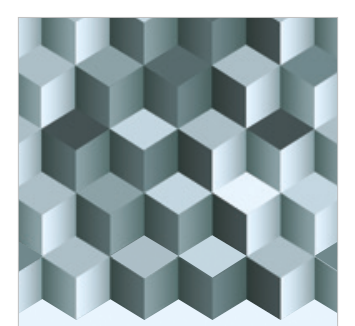

Journal of

Function Spaces

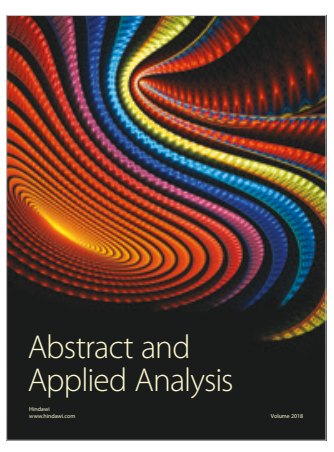

The Scientific

World Journal

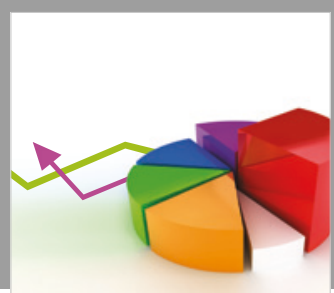

Journal of

Probability and Statistics
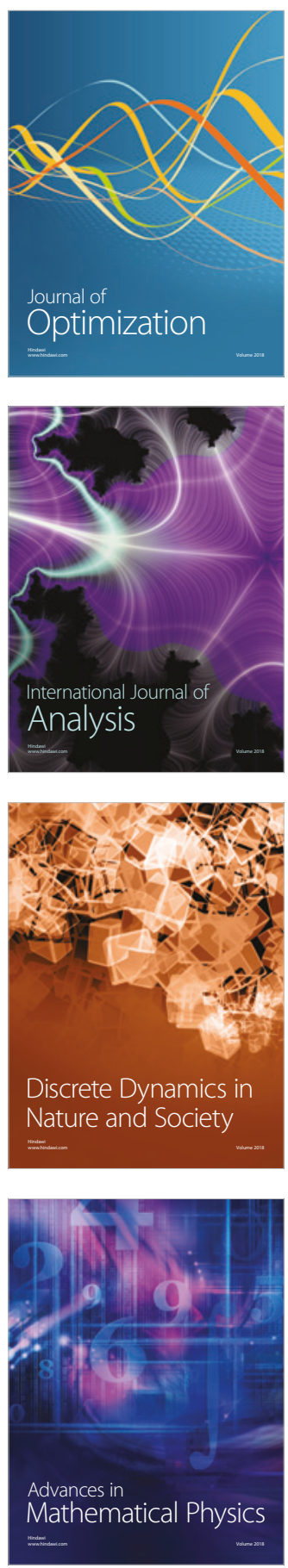